\title{
Reducing health disparities among indigenous populations: the role of collaborative approaches to improve public health systems
}

\author{
Piotr Wilk ${ }^{1,2,3} \cdot$ Martin Cooke $^{4,5} \cdot$ Saverio Stranges $^{1} \cdot$ Alana Maltby $^{1}$
}

Received: 20 July 2017 / Accepted: 29 July 2017/Published online: 18 August 2017

(c) Swiss School of Public Health (SSPH+) 2017

Indigenous peoples (First Nations, Inuit, and Métis) are the youngest and fastest growing portion of the Canadian population. In 2011, approximately 1.4 million people reported an Indigenous identity (Indigenous and Northern Affairs Canada 2013). It is well established that Indigenous peoples in Canada experience a disproportionate burden of health problems compared to the non-Indigenous population (Frohlich et al. 2006). Indigenous peoples are at greater risk for preventable chronic diseases such as type II diabetes, cardiovascular disease, and some cancers (UN Department of Economic and Social Affairs 2009). These disparities in health are not limited to Canada; they are also observed in other colonial nations such as Australia, New Zealand, and the United States (King et al. 2009; UN Department of Economic and Social Affairs 2009).

While inequalities in health among individuals and populations are a complex interplay of genetic factors, health behaviours, and socio-environmental determinants (Loppie Reading and Wien 2009), it is likely that the root

Piotr Wilk

piotr.wilk@schulich.uwo.ca

1 Department of Epidemiology \& Biostatistics, Western University, Kresge Building, Room K201, London, Ontario N6A 5C1, Canada

2 Department of Paediatrics, Western University, Kresge Building, Room K201, London, Ontario N6A 5C1, Canada

3 Child Health Research Institute, 800 Commissioners Road East, London, Ontario N6C 2V5, Canada

4 School of Public Health and Health Systems, University of Waterloo, PAS 2040, 200 University Ave West, Waterloo, Ontario N2L 3G1, Canada

5 Department of Sociology \& Legal Studies, University of Waterloo, PAS 2040, 200 University Ave West, Waterloo, Ontario N2L 3G1, Canada causes of these disparities are to be found in the structural relations between Indigenous peoples and settler societies, which perpetually disadvantage Indigenous peoples (King et al. 2009). The descendants of original inhabitants experience poorer socioeconomic conditions than do the descendants of settlers or immigrants (Cooke et al. 2007). It is necessary to consider how health behaviours are influenced by both intermediate factors (e.g., community infrastructure, resources, systems, and capacities) and distal determinants (e.g., historical, political, social, and economic contexts; Frohlich et al. 2006; Loppie Reading and Wien 2009).

Among the colonial policies in Canada, forced attendance at residential schools was particularly damaging to Indigenous peoples. The residential school system was intended to eliminate Indigenous languages, cultural traditions, and spiritual beliefs of Indigenous children to assimilate them into Euro-Canadian ways of life (Kirmayer et al. 2003; Truth and Reconciliation Commission of Canada 2015). In addition to cultural and social effects of being forcibly removed from their families and culture, many children were subjected to physical, sexual, psychological, and spiritual abuse while attending the schools (Truth and Reconciliation Commission of Canada 2015), which has had lasting consequences such as health problems, substance abuse, increased mortality and suicide rates, and families and community breakdown (Kirmayer et al. 2003). These effects are similar to those experienced in the United States and Australia, where boarding or residential schools were also a form of assimilation (King et al. 2009).

Currently, the majority of Indigenous people in Canada reside in urban areas, outside of discrete Indigenous communities (such as First Nations reserve communities) and much of the recent growth in Indigenous population has 
occurred in urban centres (Indigenous and Northern Affairs Canada 2013). In urban Canadian settings, a main challenge in improving Indigenous peoples' health is the complexity of the health systems that serve First Nations, Métis, and Inuit peoples, and the difficulty coordinating activity and mobilising resources across a large number of diverse public health system actors. Further complicating the situation, federal, provincial, and territorial jurisdictions governing Indigenous health systems and service supports differ for each cultural identity group.

Given the complex intersection and interrelation of determinants that affect the health and wellness of Indigenous populations, it is necessary to move beyond individual factors to address societal level problems, including the social and historical impact of colonization in terms of structural disadvantages and health inequalities (Frohlich et al. 2006; Loppie Reading and Wien 2009).

Cross-sector collaboration and a focus on systems thinking may be a way to address the increased risk of chronic disease among Indigenous populations, particularly those in urban areas (Wilk and Cooke 2015). Collaborative interventions in addition to addressing their short term goals, contribute to improving relationships and building trust, which is essential in developing partnerships between 'universal' and Indigenous organizations and communities (Treloar et al. 2014). Evidence from a systems-level intervention, the Healthy Weights Connection (Wilk and Cooke 2015), highlights that implications for policy and practice include the need for increased cultural awareness among 'universal' service providers, including education about the different First Nations, Métis, and Inuit communities, cultures and histories in Canada, and the impact of colonization on health. The lack of awareness and understanding among 'universal' service providers in this regard was identified as a key barrier by Indigenous organizations, which, if overcome, could foster more successful collaborations. Effective and sustainable interventions and programs should aim to change the beliefs, knowledge, actions, and institutional relationships among the various organizations in the public health system (Wilk and Cooke 2015). This model could be applied to tackle health inequalities among other marginalized populations around the globe.

\section{References}

Cooke M, Mitrou F, Lawrence D, Guimond E, Beavon D (2007) Indigenous well-being in four countries: an application of the UNDP'S human development index to indigenous peoples in Australia, Canada, New Zealand, and the United States. BMC Int Health Hum Rights 7:9

Frohlich KL, Ross N, Richmond C (2006) Health disparities in Canada today: some evidence and a theoretical framework. Health Policy 79:132-143. doi:10.1016/j.healthpol.2005.12.010

Indigenous and Northern Affairs Canada (2013) Aboriginal demographics from the 2011 National Household Survey. Statistics Canada

King M, Smith A, Gracey M (2009) Indigenous health part 2: the underlying causes of the health gap. Lancet 374:76-85. doi:10. 1016/S0140-6736(09)60827-8

Kirmayer LJ, Simpson C, Cargo M (2003) Healing traditions: culture, community and mental health promotion with Canadian Aboriginal peoples. Australas Psychiatry 11:S15-S23. doi:10.1046/j. 1038-5282.2003.02010.x

Loppie Reading C, Wien F (2009) Health inequalities and the social determinants of aboriginal peoples' health. National Collaborating Centre for Aboriginal Health, Prince George

Treloar C, Gray R, Brener L et al (2014) "I can't do this, it's too much": building social inclusion in cancer diagnosis and treatment experiences of Aboriginal people, their carers and health workers. Int J Public Health 59:373-379. doi:10.1007/ s00038-013-0466-1

Truth and Reconciliation Commission of Canada (2015) Honouring the truth, reconciling for the future: summary of the final report of the Truth and Reconciliation Commission of Canada

UN Department of Economic and Social Affairs (2009) State of the world's indigenous peoples. United Nations, New York

Wilk P, Cooke M (2015) Collaborative public health system interventions for chronic disease prevention among urban aboriginal peoples. Int Indig Policy J. doi:10.18584/iipj.2015.6. 4.3 